\title{
Personalien
}

Todesfälle / Décès / Decessi

Mansour Ghofli (1946), † 25.8.2015, Facharzt für Gynäkologie und Geburtshilfe, 5600 Lenzburg

Alfonso Andres Castano Almendral (1933), $\dagger 31.8 .2015$,

Facharzt für Gynäkologie und Geburtshilfe, 4125 Riehen

Michel Weber (1934), † 16.9.2015,

Spécialiste en chirurgie, 1223 Cologny

Hans Fellmann (1924), † 18.9.2015,

Facharzt für Kardiologie und Facharzt

für Allgemeine Innere Medizin,

6006 Luzern

Ariane Vollery-Baer (1927), † 18.9.2015,

1752 Villars-sur-Glâne
Praxiseröffnung /

Nouveaux cabinets médicaux /

Nuovi studi medici

AG

Benedikt Strub,

Facharzt für Plastische, Rekonstruktive und Ästhetische Chirurgie und Facharzt für Handchirurgie, Othmarsingerstrasse 3 , 5605 Dottikon

GE

Caroline Werner,

Fachärztin für Allgemeine Innere Medizin, 140, route d'Aïre, 1219 Aïre

GR

\section{Barbara Witte,}

Fachärztin für Kinder- und Jugendmedizin und Praktische Ärztin, Dischmastrasse 63, 7260 Davos Dorf

LU

Cordula Zwinggi,

Fachärztin für Kinder- und Jugendmedizin, Landenbergstrasse 3b, 6005 Luzern

\section{ZH}

\section{Reto Meuli,}

Facharzt für Anästhesiologie, Winkelwiese 8, 8637 Laupen ZH

Stefan Teske,

Facharzt für Dermatologie und Venerologie, Dermatologische Praxis am Hauptbahnhof, Bahnhofstrasse 110, 8001 Zürich

\section{Aargauischer Ärzteverband}

Zur Aufnahme als ordentlich praktizierende Mitglieder in den Aargauischen Ärzteverband haben sich angemeldet:

Karen Burger, 5745 Safenwil,

Praxiseröffnung in Praxisgemeinschaft in Oftringen seit 1. September 2015

Arne-Carsten Göbel, 5745 Safenwil, Praxiseröffnung in Praxisgemeinschaft in Oftringen seit 1. September 2015

Monika Donath, 5200 Brugg, Fachärztin für Dermatologie und Venerologie, Praxiseröffnung in Praxisgemeinschaft in Brugg per 1. Oktober 2015

Gabriela Meister, 5722 Gränichen, Fachärztin für Gynäkologie und Geburtshilfe, spez. Operative Gynäkologie und Geburtshilfe, Praxiseröffnung in Aarau per 1. Januar 2016

Ivan Stamenic, 6044 Udligenswil, Facharzt für Innere Medizin, Facharzt für Gastroenterologie, FMH, Praxiseröffnung in Praxisgemeinschaft in Wettingen per 1. März 2016

Als Leitender Arzt hat sich angemeldet:

Florian Schleich, 8083 Zürich, Facharzt für Radiologie, Leitender Arzt im Spital Muri in Muri seit 1. März 2015

Diese Kandidaturen werden in Anwendung von Art. 5 der Statuten des Aargauischen Ärzteverbandes veröffentlicht. Einsprachen müssen innert 14 Tagen seit der Bekanntmachung schriftlich und begründet der Geschäftsleitung des Aargauischen Ärzteverbandes eingereicht werden. Nach Ablauf der Einsprachefrist entscheidet die Geschäftsleitung über Gesuch und allfällige Einsprachen. 
Ärztegesellschaft des Kantons Bern

Ärztlicher Bezirksverein Bern Regio

Zur Aufnahme als ordentliches Mitglied hat sich angemeldet:

Lars Diedrichsen, Facharzt für Radiologie, Klinik Sonnenhof, Buchserstrasse 30, 3006 Bern

Einsprachen gegen dieses Vorhaben müssen innerhalb 14 Tagen seit der Veröffentlichung schriftlich und begründet beim Präsidenten des Ärztlichen Bezirksvereins Bern Regio eingereicht werden. Nach Ablauf der Frist entscheidet der Vorstand über die Aufnahme des Gesuchs und über die allfälligen Einsprachen.
Ärztegesellschaft des Kantons Schwyz

Zur Aufnahme in die Ärztegesellschaft des Kantons Schwyz hat sich angemeldet:

René Knollmann, Facharzt für Allgemeine Innere Medizin, Chefarzt Innere Medizin Regionalspital Einsiedeln ab 1.10.2015.

Einsprachen gegen diese Aufnahme richten Sie schriftlich innert 20 Tagen an Dr. med. Hugo Brunner, Dorfstrasse 14, 6417 Sattel.

\section{Ärztegesellschaft Thurgau}

Zum Eintritt in die Ärztegesellschaft Thurgau hat sich gemeldet:

Thomas Holzbach, Ringstrasse 13, 8500 Frauenfeld
Preise / Prix / Premi

Krebspreis und Anerkennungspreis Der mit 10000 Franken dotierte Krebspreis geht an Prof. Dr. med. Monica Castiglione für ihr unermüdliches Engagement, das sie während Jahrzehnten sowohl als Brustkrebsexpertin an den Universitäten Bern und Genf wie auch als Direktorin der "International Breast Cancer Study Group» (IBCSG) und des Schweizerischen Instituts für angewandte Krebsforschung (SIAK) an den Tag gelegt hat. Monica Castiglione hat sich auf politischer und wissenschaftlicher Ebene sowie in der Öffentlichkeit stets für die Ziele der Krebsliga stark gemacht. Den mit 5000 Franken dotierten Anerkennungspreis verleiht die Krebsliga Schweiz an Martin Rothenbühler. Der Gründer und langjährige Geschäftsleiter der Stiftung sanaCERT ist der Krebsliga sozusagen als Geburtshelfer beigestanden, als sie das Qualitätslabel für Brustzentren vor vier Jahren ins Leben gerufen hat. Das Label ist seither schon zwölf verschiedenen Zentren in der Schweiz verliehen worden. 